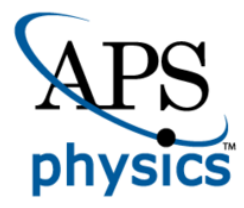

This is the accepted manuscript made available via CHORUS. The article has been published as:

\title{
Tunable helium bubble superlattice ordered by screw dislocation network
}

Zengfeng Di, Xian-Ming Bai, Qiangmin Wei, Jonghan Won, Richard G. Hoagland, Yongqiang Wang, Amit Misra, Blas P. Uberuaga, and Michael Nastasi

Phys. Rev. B 84, 052101 - Published 24 August 2011

DOI: 10.1103/PhysRevB.84.052101 


\section{Tunable helium bubble superlattice ordered by screw dislocation network}

Zengfeng Di, ${ }^{1,3,}$ Xian-Ming Bai, ${ }^{2}$ Qiangmin Wei, ${ }^{1}$ Jonghan Won, ${ }^{2}$ Richard G. Hoagland, ${ }^{2}$

Yongqiang Wang, ${ }^{2}$ Amit Misra, ${ }^{1}$ Blas P. Uberuaga, ${ }^{2}$ and Michael Nastasi ${ }^{1}$

${ }^{1}$ Materials Physics and Applications Division, MPA-CINT, Los Alamos National Laboratory, Los Alamos, New Mexico, 87545, USA

${ }^{2}$ Materials Science and Technology Division, MST-8, Los Alamos National Laboratory, Los

Alamos, New Mexico, 87545, USA

${ }^{3}$ State Key Laboratory of Functional Materials for Informatics, Shanghai Institute of Microsystem and Information Technology, Chinese Academy of Sciences, Shanghai 200050, China 


\begin{abstract}
Helium bubble nucleation at low-angle twist boundaries in gold has been investigated. It is found that the helium bubbles preferentially nucleate at screw dislocation nodal points and result in helium bubble superlattice formation, which is completely isomorphic with the screw dislocation network along the twist grain boundary. Molecular statics calculations reveal that defect formation/solution energies along the screw dislocations, especially at the nodal points, are lower than their bulk counterparts. It is believed that this driving force is responsible for the helium bubble superlattice formation. Our study suggests that grain boundary engineering via adjustable twist angles in parallel boundaries to form tunable 3-D bubble superlattices could afford a very promising approach for design of radiation tolerant materials.
\end{abstract}

PACS: 61.72Mm; 61.72.J-; 61.72.Lk; 61.50.Lt

Keywords: Grain boundaries; Point defects; Dislocation; Crystal bonding

* Corresponding author:

Dr. Zengfeng DI

Telephone: 86-21-62511070

Fax: 86-21-52419931

Electronic mail: dizengfeng@,hotmail.com 
The demand to reduce reliance on fossil fuels while meeting rapidly growing energy needs has renewed interest in nuclear energy - both fission and fusion. High energy particles in nuclear power environments induce displacements of large numbers of atoms in structural materials from their lattice positions, creating excess defects (vacancies and self-interstitial atoms) and generating insoluble helium from transmutation reactions. The combination of helium and defects leads to a microstructural evolution in which helium bubbles often form and contribute to both swelling and embrittlement, degrading the properties of fuel cladding and first wall materials of nuclear reactors $[1,2]$. However, recent proposals have suggested that helium bubbles could be managed, thereby transforming them from a liability into an asset in controlling damage evolution in irradiated materials [1,3].Odette and Hoelzer [1]propose that nanometer scale helium bubbles can act as sinks for point defects, providing enhanced immunity to radiation damage. It has also been shown that interfaces, phase boundaries and grain boundaries can mitigate radiation defects and influence helium bubble nucleation and growth [4-8]. In many cases, this has been attributed to the presence of dislocations that make up the interface structure[9-11].

Based on these previous findings it appears that the important consideration in helium management at interfaces is to understand the role of interface structure on helium bubble nucleation and growth. In this work, we create well-defined grain boundaries in gold, i.e., pure twist boundaries, with precisely controlled twist angles forming a well-defined screw dislocation network. We investigate the role of grain boundary structure on the nucleation and growth of helium bubbles. We observe that helium bubbles preferentially nucleate at the nodes of the screw dislocation network, forming a helium bubble superlattice. Molecular statics calculations suggest that bubble formation is associated with lower vacancy formation energy and lower He solution energies within the screw dislocations and dislocation nodes.

Single crystal (001) Au films, $50 \mathrm{~nm}$ in thickness, were deposited on 600nm Ag single crystal layers, which had been deposited on polished $\mathrm{NaCl}$ substrates by means of electron beam evaporation. The deposition temperatures were set at $350{ }^{\circ} \mathrm{C}$ and $50{ }^{\circ} \mathrm{C}$ for $\mathrm{Ag}$ and $\mathrm{Au}$, respectively, and the deposition rate was $2 \AA / \mathrm{s}$. The $50 \mathrm{~nm}-50 \mathrm{~nm}$ Au-Au twist boundary was obtained by welding two $\mathrm{Au} / \mathrm{Ag} / \mathrm{NaCl}$ assemblies together with a 1 degree twist with respect to each other around their common normal, i.e., [001] orientation. After welding, the $\mathrm{NaCl}$ substrates 
were dissolved in water, and the $\mathrm{Ag}$ buffer layers were etched away by $20 \% \mathrm{HNO}_{3}$ solution. The remaining free-standing Au bicrystal specimen was placed on a copper grid, and implanted at 250 ${ }^{\circ} \mathrm{C}$ with a fluence of $5 \times 10^{15} \mathrm{~cm}^{-2} \mathrm{He}$ ions at an energy of $22.5 \mathrm{keV}$. The implantation energy was chosen ensure that all implanted He ions remained within the Au bicrystal and the maximum concentration of implanted He coincided with the position of the twist boundary. The implantation temperature was chosen to assist in helium bubble formation and with consideration of the bubble-deduded zone as discussed later. For comparison, a $100 \mathrm{~nm}$ thick single crystal Au foil was subjected to an identical implantation. The samples before and after He implantation were examined without any additional sample thinning or ion milling with transmission electron microscopy (TEM) using a FEI Tecnai F30 operated at $300 \mathrm{kV}$.

As shown in Fig. 1, a square grid of screw dislocations with a periodicity of $15 \mathrm{~nm}$ is created along the bonding interface of the Au bicrystal. According to Frank's law,[12] the spacing between the screw dislocations, $\Lambda$, is related to the misorientation angle, $\Theta$ :

$$
\Lambda=\frac{b}{2 \sin (\Theta / 2)}
$$

where $\mathrm{b}$ is the Burgers vector $\left(\mathrm{b}=\mathrm{a}_{\mathrm{Au}} / 2<110>\right)$. Using $\Lambda=15 \mathrm{~nm}$ (as measured from our sample) and $\mathrm{b}=0.288 \mathrm{~nm}$, the corresponding misorientation angle is calculated as 1.1 degrees, which is close to the intended 1-degree misorientation angle.

Figure 2(a) illustrates the association between helium bubbles and the nodes of the screw dislocation grid. It is clear that helium bubbles are formed exactly at the nodal points of the dislocation network, while no detectable helium bubbles are observed in the matrix. By duplicating the symmetry and alignment of the dislocation network, all observable helium bubbles are assembled two-dimensionally into a bubble superlattice at the grain boundary plane with areal density of $6 \times 10^{11} \mathrm{~cm}^{-2}$. The sizes of the helium bubbles are relatively uniform, with an average radius of about $1.2 \mathrm{~nm}$. In comparison, as shown in Fig.2 (b), randomly distributed helium bubbles with an areal density of $2 \times 10^{12} \mathrm{~cm}^{-2}$ are formed in the single crystal gold foil subjected to identical implantation conditions. The suppression of helium bubble formation in the Au bicrystal is believed to be correlated with the grain boundary. Similar as interfaces in nanolayered 
composites [7], the grain boundary, which has large excess free volume and higher diffusivity of point defects, provides inexhaustible sinks for radiation-induced point defects and a catalyst for efficient Frenkel pair annihilation. Fewer vacancies residual in the Au bicrystal retard the helium bubble nucleation subsequently. TEM focusing experiments and the size variation of helium bubbles suggest they are distributed at different depths throughout the pure Au foil; they are clearly larger than those that form at the nodes of the screw dislocation network. Comparison of Figs. 2(a) and 2(b) indicates that in the twist GB all helium bubbles are formed at the same depth i.e., the plane of twist grain boundary. Otherwise, the TEM image in Fig. 2(a) would show a similar random distribution of He bubbles as in the single crystal case.

To understand the correlation between He bubble formation and the grain boundary structure, molecular statics was used to calculate the vacancy formation energy, $\mathrm{E}_{\mathrm{vac}}$, and the $\mathrm{He}$ substitutional and interstitial solution energies for various atomic sites in a model of a twist boundary. These calculations were performed on $\mathrm{Cu}$, rather than $\mathrm{Au}$, as $\mathrm{Cu}$ has the same face-centered cubic (FCC) structure as Au and, more importantly, well established interatomic potentials exist for the $\mathrm{Cu}-\mathrm{He}$ system. We expect that our results on $\mathrm{Cu}$ give qualitative insight into the behavior in Au. Further, as we will show, calculation of vacancy behavior near twist boundaries in $\mathrm{Cu}$ shows very similar behavior in $\mathrm{Au}$. In these calculations we used the embedded atom method (EAM) potentials developed by Voter et al. for $\mathrm{Cu}-\mathrm{Cu}[13]$ and by Wang et al. for $\mathrm{Cu}-\mathrm{He}$ interactions [14]. The twist grain boundaries were created by rotating two perfect $\mathrm{Cu}$ crystals around their [100] axes, with (100) planes parallel to the formed boundary. The rotation angle between the two grains was $8.17^{\circ}$, chosen to reduce the model size. As the twist angle is still relatively small, resulting in a boundary structure that is still composed of an intersecting screw dislocation network, this boundary reproduces all of the essential features that would be present in the $1^{\circ}$ boundary examined in the experiments. The resulting dimensions of the model were $5.07 \mathrm{~nm}$ $\times 5.07 \mathrm{~nm} \times 2.89 \mathrm{~nm}$, containing $6304 \mathrm{Cu}$ atoms. Periodic boundary conditions were used in all directions. In order to find the minimum GB energy structure, one grain was shifted relative to the other grain along a $21 \times 21$ point grid at the grain boundary plane. At each point, a conjugate gradient energy minimization method was used to minimize the GB structure. The GB structure used in subsequent calculations is the minimum-energy structure from this energy surface, i.e., 
gamma surface, mapping. The spacing between dislocations within the network is $1.79 \mathrm{~nm}$, in agreement with Eq. (1) using $\Theta=8.17^{\circ}$ and $b=0.256 \mathrm{~nm}$. To calculate the vacancy formation energy as a function of position near the GB, every atom within the system was removed, one at a time, and the energy of the resulting structure was minimized. Fig. 3(a) shows the formation energy of the vacancy near the twist GB, relative to the formation energy in perfect crystal $\mathrm{Cu}-\mathrm{a}$ negative energy indicates a more favorable site for vacancy formation. Similarly, the relative solution energy of $\mathrm{He}$ as a substitutional species (replacing $\mathrm{Cu}$ atoms) and as an interstitial species were calculated, as shown in Fig. 3(b) and Fig. 3(c), respectively. Interstitial sites were identified by the method of Jiang et al.[15]

The results show the relation between the defect formation/solution energies and the structure of the twist boundary. In all cases the energies are lower at the cores of the screw dislocations than in regions away from the GB. Moreover, the energies are significantly lower within the nodes of the dislocation network. Near the center of each grid where the screw dislocation fields cancel, the vacancy and solution energies are nearly the same as perfect crystal values. Within the nodal regions, the vacancy formation energy is about $1.16 \mathrm{eV}$ lower than the value in perfect $\mathrm{Cu}, 1.27$ $\mathrm{eV}$. Thus, the vacancy formation energy at nodal points is nearly zero indicating that vacancies can easily form at the nodes. This extremely low vacancy formation energy at the intersection point of two screw dislocations is consistent with the calculations by Nomura et al. [16]. The vacancy formation energy within the core of a screw dislocation is about $0.6 \mathrm{eV}$ lower than the bulk value. The decrease in formation energy of the vacancy implies a lower He diffusion activation energy $E_{a}$ when diffusion is via a vacancy mechanism, since $E_{a}$ is the sum of the migration energy and the formation energy of the mediating defect. In this case, the migration energy of the vacancy is either unchanged or lowered near the boundary. Similar calculations on a twist boundary in Au find very similar behavior: the vacancy formation energy at the nodes is 0.97 $\mathrm{eV}$ lower than the bulk value while it is about $0.46 \mathrm{eV}$ lower at the screw dislocation cores. This gives us confidence that the calculations on $\mathrm{Cu}$ are representative of the behavior in $\mathrm{Au}$.

Consistent with our calculations, dimer calculations in alpha-iron indicate that vacancies lower their energy by moving toward a screw dislocation where they become strongly trapped [17]. Once trapped in the core region of the screw dislocation, the vacancy can migrate along the 
dislocation line with a lower migration energy until it interacts with another defect such as with He or other vacancies; the migration energy of a vacancy along the core of a screw dislocation in alpha-iron was found to be about half of that in the bulk [17]. These results along with the information presented in Fig 3(a) suggest that the clustering of vacancies will be highly probable along the screw dislocations and even more so at the intersections, leading to the formation of bubbles, which are comprised of vacancy clusters and helium atoms.

Helium bubble formation as shown in Fig 2(a) requires an agglomeration of He at nodal points. Helium diffusion could occur via either a vacancy (the dominant mechanism at equilibrium) or interstitial (possibly transiently during irradiation) mechanisms. Therefore, we investigate the solution energies of both substitutional and interstitial He to see whether the dislocation network induces He segregation. The relative solution energy of substitutional He near the twist GB is shown in Fig. 3(b), revealing a pattern that is similar to that of the vacancy [Fig. 3 (a)], although the magnitude of the segregation energy is much smaller. The formation energy of substitutional $\mathrm{He}$ is lower in the cores of the screw dislocations and lowest at the nodal points, compared to the perfect crystal. The maximum reduction in the substitutional He solution energy is about $0.38 \mathrm{eV}$ at the nodal points of the screw dislocation network while it is about $1.16 \mathrm{eV}$ for vacancy formation. As the structure of He interstitials is more complex than substitutional He, the interstitial He solution energy map [Fig. 3(c)] does not precisely mirror the atomic structure of the grain boundary, but still exhibits the general features of the screw dislocation network. As with the other defects, the interstitial He solution energies are lower at sites near the screw dislocation cores and nodal points than in the interior regions of the grids. The minimum formation energy of interstitial $\mathrm{He}$ is at the nodal region, and is $0.92 \mathrm{eV}$ lower than in perfect crystal.

The data in Fig 3 suggest that: (1) vacancies have an energetic preference to reside in the screw dislocation lines and nodes, particularly at the nodal points; and (2) regardless of the form it adopts (interstitial or substitutional), He also prefers to reside at the nodal points in the dislocation network. Thus, there are thermodynamic driving forces for $\mathrm{He}$ to be at the nodal points, which favors these as nucleation sites for He bubbles. 
Nanostructured ferritic alloys (NFAs) have received a great deal of attention because of their excellent mechanical properties and resistance to irradiation damage. NFAs are Fe alloys with nanometer-sized oxide particles dispersed in the matrix. Recently, it has been proposed that one reason NFAs exhibit such high radiation tolerance is due to the formation of dispersed He bubbles at the interfaces between the oxide particles and the matrix. Normally, He is viewed as a liability, as the formation of He bubbles that grow without bound lead to swelling of the material. In NFAs, the high density of oxide particles results in a high density of nanometer-sized He bubbles. These dispersed bubbles are excellent sinks, not only for He, but irradiation-induced defects (interstitials and vacancies) because they act nearly like a perfect surface. As a result, NFAs transform He from a liability to an asset, increasing the radiation tolerance of the material $[1,3]$.

In the present work, we also obtain a large concentration of nanometer-sized He bubbles, formed on the intersections of the screw dislocation network of the twist boundary. Thus, by changing the twist angle, one can control the density of He bubbles and thus the overall radiation tolerance of the material. Clearly, in a real material, one twist boundary is not going to provide a sufficient density of He bubbles, but if a three-dimensional dislocation network can be built into the material, a high density of dispersed He bubbles could be achieved even in a single component material such as $\mathrm{Au}$, again transforming $\mathrm{He}$ from a liability to an asset and providing enhanced radiation tolerance.

To ensure the maximal capture of He into the nodes of the dislocation network, the spacing between those nodes must be controlled. That means that the spacing must be smaller than twice the width of the bubble-denuded zones often observed near boundaries in irradiated materials [10]. If the distance between nodes within the three-dimensional network is smaller than the He-node interaction distance, all $\mathrm{He}$ will eventually become trapped at nodes. Using the relationship proposed by Weeks et al.[18], the width of the bubble-denuded zone can be estimated:

$$
\begin{aligned}
& r^{*}=\left[\frac{20}{\pi^{2}} \frac{D_{v}}{k T} \Omega G b^{2}\left(\frac{1-v}{7-5 v}\right) t\right]^{\frac{1}{4}} \\
& D_{v}=D_{0} \exp \left(-\frac{E_{a}}{k T}\right)
\end{aligned}
$$


where $D_{v}$ is the volume diffusion coefficient, $D_{0}$ is the pre-exponential coefficient, $E_{a}$ is the activation energy for diffusion, $\mathrm{k}$ is Boltzmann's constant, $\mathrm{T}$ is absolute temperature of helium implantation, $\Omega$ is the atomic volume, $\mathrm{G}$ is the shear modulus of the matrix, $\mathrm{b}$ is the Burgers vector of the screw dislocation, $v$ is Poisson's ratio, and t is the implantation duration, respectively. Using values corresponding to the $\mathrm{Au}$ system $\left(\mathrm{D}_{0}=0.091 \mathrm{~cm}^{2} / \mathrm{s}[19] ; \mathrm{E}_{\mathrm{a}}=0.82 \mathrm{eV}[19] ; \mathrm{k}=1.38 \times 10^{-23} \mathrm{~J} / \mathrm{K}\right.$; $\Omega=1.18 \times 10^{-23} \mathrm{~cm}^{3} ; \mathrm{G}=27 \mathrm{Gpa} ; \mathrm{b}=2.88 \times 10^{-8} \mathrm{~cm}$ and $v=0.42$ ), the radius of the bubble-denuded zone for the twist grain boundary is plotted against irradiation temperature and irradiation time in Fig. 4. In general, the bubble-denuded zone increases with increasing irradiation temperature and irradiation time. At low temperature where vacancies, and thus He, are relatively immobile, the bubble-denuded zone is barely observed. However, it rapidly increases at high temperature. The twist grain boundary could even attract vacancies or helium a half micron away when the irradiation temperature is $700 \mathrm{~K}$. Using values consistent with our experimental conditions, i.e., $\mathrm{T}=523 \mathrm{~K}$ and $\mathrm{t}=1 \mathrm{~h}$, we calculate $\mathrm{r}^{*}=140 \mathrm{~nm}$, which is close to experimentally observed denuded zones $(\sim 100 \mathrm{~nm})$ in $\mathrm{Al}$ at similar homologous temperatures [20]. In a material in which a three-dimensional node network is formed from layered twist boundaries, the spacing between those boundaries must be no greater than $2 r^{*}$, as demonstrated in the insert of Fig. 4. Such architecture of twist boundaries may be promising for the design of radiation tolerant materials.

In conclusion, we observe preferential helium bubble formation at the nodes of screw dislocations formed along a twist boundary in Au. The screw dislocation network provides a perfect template for helium bubble formation, resulting in a superlattice of helium bubbles coincident with the nodal structure of the dislocation network. Molecular statics calculations indicate that lower vacancy formation and substitutional He and interstitial He solution energies along the screw dislocations provide the driving force for the segregation of vacancies and helium atoms towards the screw dislocation, thus resulting in He bubble nucleation at dislocation nodes to form the bubble superlattice.

\section{Acknowledgements}


The experimental portion of this work was sponsored by the Laboratory Directed Research and Development (LDRD) program at Los Alamos National Laboratory under Project No. 20090061DR. The modelling work was supported as part of the Center for Materials at Irradiation and Mechanical Extremes, an Energy Frontier Research Center funded by the U.S. Department of Energy, Office of Science, Office of Basic Energy Sciences under Award Number 2008LANL1026. 
References:

[1] G.R.odette and D. T. Hoelzer, JOM 62, 84 (2010).

[2] S. J. Zinkle and J. T. Busby, Materials Today 12, 12, (2009)

[3] G. R. Odette et al., Annu. Rev. Mater. Res. 38, 471 (2008)

[4] M. J. Demkowicz et al., Phys. Rev. Lett. 100, 136102 (2008)

[5] A. Misra et al., JOM 59, 62 (2007)

[6] N. Nita et al., J. Nucl. Mater. 329-333, 953 (2004)

[7] T. Shen et al., Appl. Phys. Lett. 90, 263115 (2007)

[8] X. -M. Bai et al., Science 327, 1631 (2010)

[9] G. W. Greenwood et al., J. Nucl. Mater. 4, 305 (1959)

[10] P. L. Lane and P. J. Goodhew, Phil. Mag. A 48, 965 (1983)

[11] B.N. Singh et al., J. Nucl. Mater 125, 287(1984)

[12] F. C. Frank, Carnegie Institute of Technology Symposium on the Plastic Deformation of Crystalline Solids, Office of Naval Research 1, 150 (1950)

[13] A. F. Voter, Los Alamos Unclassified Technical Report \#LA-UR 93-3901 (1993)

[14] L. Wang and X. J. Ning, Chin. Phys. Lett. 20, 1416 (2003)

[15] C. Jiang, et al., Scr. Mater. 58, 739 (2008)

[16] M. Nomura and J. B. Adams, J. Mater. Res. 7, 3202 (1992)

[17] H. L. Heinisch et al., Philos Mag. 90, 885 (2010)

[18] R. W. Weeks et al., Acta. Metallurgica 17, 1403 (1969)

[19] P. Shewmon, Diffusion in Solids, 2nd ed. (TMS Press, Warrendale, PA, 1989)

[20] B.N. Singh et al., J. Nucl. Mater. 122, 703 (1984) 
Figure caption

Fig.1. a square network of screw dislocations in a (001) twist grain boundary with a misorientation angle $\Theta \approx 1^{\circ}$.

Fig.2. Plan view of specimens with $22.5 \mathrm{keV} 5 \times 10^{15} \mathrm{~cm}^{-2} \mathrm{He}$ implantation at $250{ }^{\circ} \mathrm{C}$ : (a) $\mathrm{Au}$ bicrystal; (b) pure Au foil.

Fig.3. Formation and solution energies relative to the bulk for different atom sites along (001) $8.17^{\circ}$ twist boundary of $\mathrm{Cu}$ bicrystal (a) pure $\mathrm{Cu}$ vacancy; (b) substitutional $\mathrm{He}$; (c) interstitial He. Only a portion of the calculated twist GB plane is shown in each figure for clarity. The color of each defect site represents their respective defect formation energy. Note a value of zero in each figure represents the respective energy in the bulk region.

Fig. 4. The radius of bubble-denuded zone for twist grain boundary in gold vs. irradiation temperature and irradiation time. The proposed three-dimensional screw dislocation network is inserted as well. 


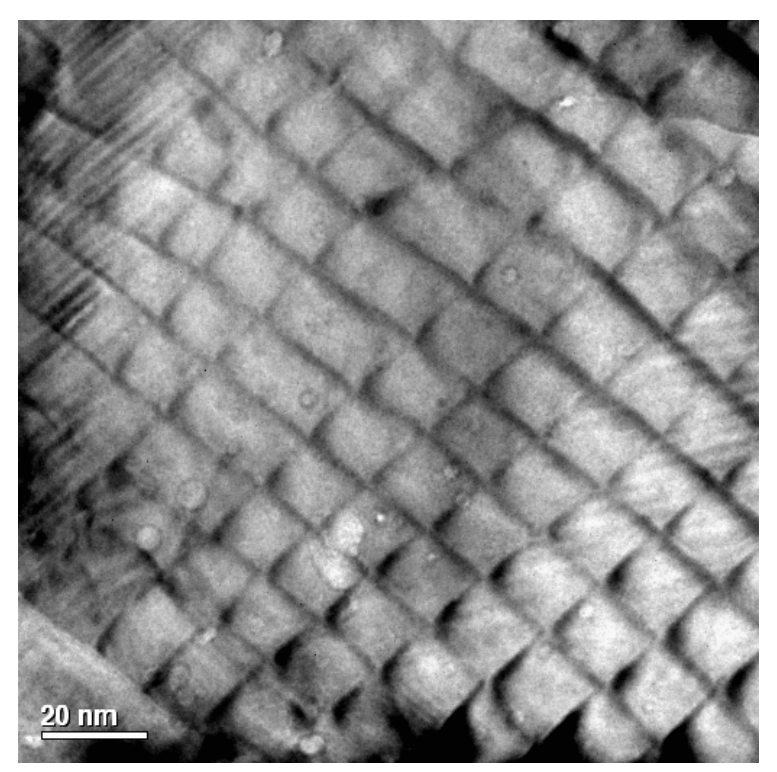

Fig. 1 

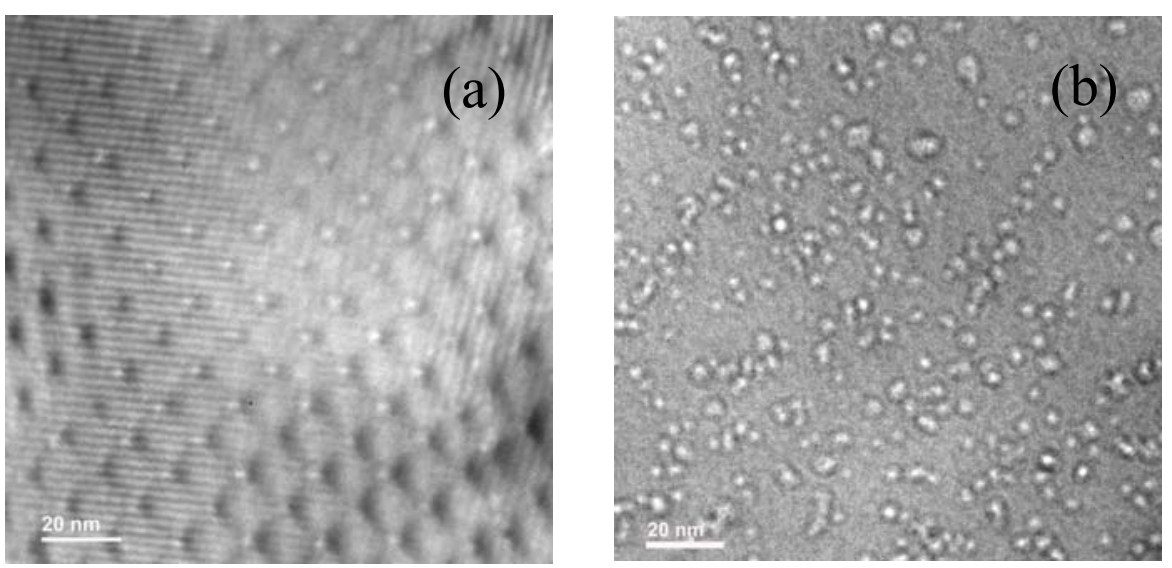

Fig. 2 


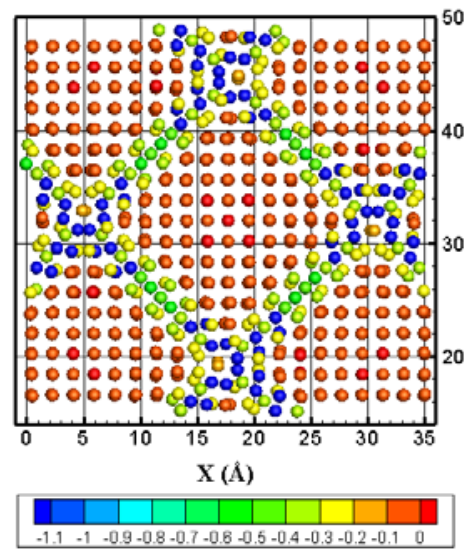

(a)

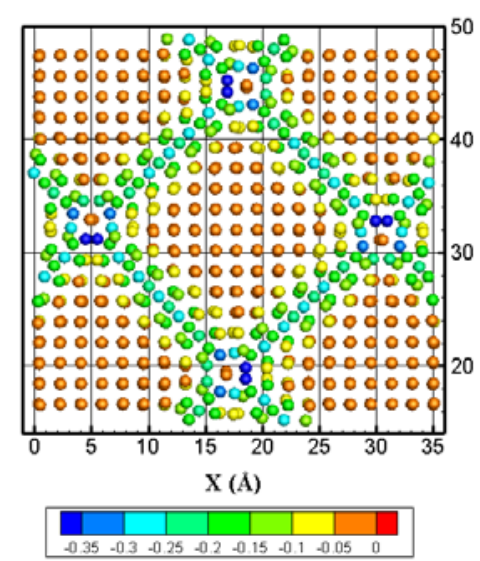

(b)

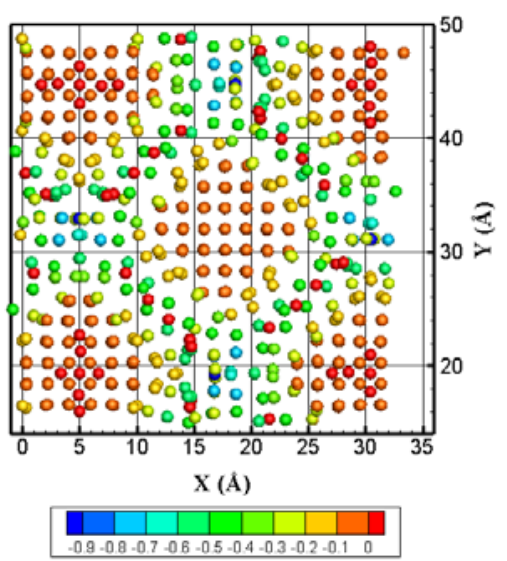

(c)

Fig. 3 


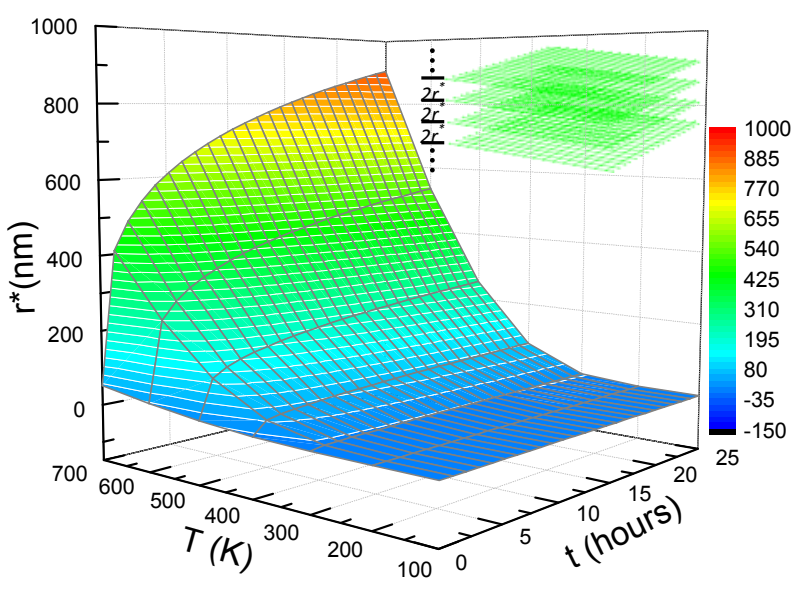

Fig. 4 